12 BARRIERS AND FACILITATORS TO USING REAL-WORLD DATA IN HEALTHCARE SETTINGS IN LOW-AND MIDDLEINCOME COUNTRIES: A SYSTEMATIC REVIEW OF QUALITATIVE STUDIES

1,2Ranin Soliman, ${ }^{2}$ Nia Roberts, ${ }^{1}$ Alaa Elhaddad, ${ }^{1}$ Wael Eweida, ${ }^{2}$ Jason Oke, ${ }^{2}$ Carl Heneghan. ${ }^{1}$ Children's Cancer Hospital 57357 - Egypt, Cairo, Egypt; ${ }^{2}$ University of Oxford, Oxford, UK

\subsection{6/bmjebm-2019-EBMLive.93}

Objectives To determine the barriers and facilitators to using real world data (RWD) in healthcare settings in low-and middle-income countries (LMICs).

Method We conducted a systematic review through searching MEDLINE, EMBASE, Global Health Database and CINAHL. We searched for qualitative studies that address the use of RWD, barriers and facilitators, and its applications in LMICs. Study participants included healthcare settings/organizations in LMICs which collect and use RWD. Primary outcomes are the roles of using RWD, and barriers/facilitators affecting the applications of RWD in healthcare settings in LMICs. Data extraction included contextual and methodological data. Quality of review will be assessed using the CASP Qualitative checklist, and we will follow the ENTREQ checklist for synthesis of qualitative research. Risk of bias will be assessed using GRADE-CERQual to determine the level of confidence from qualitative evidence synthesis. Qualitative data synthesis will be done as narrative/ descriptive synthesis of the roles of using RWD in healthcare settings in LMICs; thematic synthesis and conceptual framework of the barriers/facilitators of using RWD and its applications.

Results The review addressed the use of real-world data in healthcare settings in LMICs, according to World Bank classification including 137 countries as per World Bank country classification. Initial search across the four databases showed a total of 2,245 search results. The results were separated into three sets; systematic reviews $(n=27)$ from 2012 until 2018; primary studies $(\mathrm{n}=2,048)$ from 1988 until 2019; and conference abstracts $(n=170)$ from 2004 until 2018. Preliminary searches are completed, and piloting of the study selection process was done. Formal screening of results against eligibility criteria is underway and will be presented in conference.

Conclusions The use of RWD in healthcare settings in LMICs is important to make evidence-based improvements in care delivery and health outcomes. Results from this systematic review will address the gap in evidence about what real world data is used in LMICs and the barriers and facilitators to its use. This review will generate qualitative evidence about the roles, barriers and facilitators and real-world applications of using RWD in healthcare settings in LMICs.

\section{THE SCIENTIFIC INDEX OF INTEGRITY AMONG AUTHORS OF ARTICLES PUBLISHED IN MAJOR JOURNALS: A NEW METRIC FOR EVALUATING THE SCIENTIFIC ECOSYSTEM}

\footnotetext{
1,2,3 ${ }^{3}$ Luis Correia, ${ }^{2}$ Diego Rabelo, ${ }^{4}$ Alessandra Caldas, ${ }^{2}$ Bruno Góes, ${ }^{4}$ Daniel Medina, ${ }^{5} J a n i n e ~ M a g a l h a ̃ e s,{ }^{5}$ Denise Matias. ${ }^{1}$ Bahiana Medical and Public Health School, Salvador, Brazil; ' ${ }^{2}$ Center for Evidence Based Medicine, BAHIANA, Salvador, Brazil; ${ }^{3}$ Hospital São Rafael, D'Or Institute for Research and Education, Salvador, Brazil; ${ }^{4}$ Federal University of Bahia, Santo Antônio de Jesus, Brazil, ${ }^{5}$ Center for Evidence Based Medicine - BAHIANA, Salvador, Brazil
}

10.1136/bmjebm-2019-EBMLive.94
Objectives Before proved by evidence, plausible hypotheses are more likely to be false than true. Therefore, one should expect more true negative studies than true positive studies. However, mechanisms such as imprecision, bias in study design, outcome reporting bias, spin and publication bias fabricates a higher prevalence of positive studies. Scientific integrity constitutes a series of mechanisms to prevent such bias towards positive results. Based on this rational, a higher number of negative studies in comparison with positive studies indicates scientific integrity of an author, laboratory, scientific field or journal.

To propose the 'index of integrity' and use it to describe scientific integrity among first authors of papers published in major journals.

Method We selected the first authors of any original article published during the first month of 2019 in three major medical journals: Journal of American Medical Association, The Lancet and British Medical Journal. Lifetime articles published by these individuals as first authors in Pubmed indexed journals were selected and defined as positive or negative according to abstract conclusion.

A positive article was defined as those with (1) a positive conclusion based on the primary analysis or (2) a negative primary conclusion spun into positive by emphasis on secondary positive results. Otherwise, the article was defined as negative. The index of integrity was calculated by the ratio between negative articles (numerator) and positive articles (denominator). An index $>1$ is desired as a marker of scientific integrity.

Results A total of 27 authors were selected, with a median of 5 articles (interquartile range $=1-7$, minimum of 1 and maximal of 55) published during lifetime in Pubmed indexed journals. The prevalence of positive articles was $87 \%$ (95\% CI $=82 \%-91 \%)$ and the utilization of spin to positivize the conclusion was present in $7.9 \%$ of positive articles.

Sixteen authors (59\%) had no negative studies and leading to an index of integrity equal to zero. Only 4 authors (15\%) had an index higher than 1 . The average estimation for the index of integrity was 0.40 (95\% CI $=0.10-0.70)$. There was no association between the index and total number of publication or the practice of spin.

Conclusions In this preliminary report, an especially higher number of positive articles compared with negative articles suggests undesired level of scientific integrity among first authors of major publications in medical science. The index of integrity, first utilized in this report, should be validated in larger samples of authors.

\section{EVIDENCE BASED MEDICINE: MYTH OF INCORPORATION OF NOVEL IDEAS IN COMMON PRACTICE

\footnotetext{
${ }^{1,2}$ Archana Raysee, ${ }^{1}$ Muhammad Junaid Saleem. ${ }^{1}$ Medway Maritime Hospital, Kent, UK; ${ }^{2}$ University of Edinburgh, Edinburgh, UK
}

\subsection{6/bmjebm-2019-EBMLive.95}

Objectives EBM is the best method to practice medicine. But does this quote a scenario of a perfect world as the only way it can be guaranteed is theoretically. As argued sometimes that day to day practice based on clinical expertise and experience may bring results not so different from the evidence proven methods. 
Also, it is apparent that the gap in generating evidence and practicing medicine is getting wider to cover, as most of the efforts are put into trials to establish superiority of one method over another rather than improving quality of care by striving to incorporate it in daily practice. 1

The main objectives are to assess the obstacles in the process of incorporation of EBM in day to day practice starting from lack of realisation of right question to challenge old and outdated practice to the development of strong evidence and implementation in order to improve clinical practice.

Method A case base discussion was performed with Trainees and Special Doctors in the field of Urology.

The literature search was performed on Cochrane, Pubmed and Google scholar to identify the role of leadership in EBM and accessibiltiy of under training doctors to understand, comprehend and implement the need of practicing Evidence Based Medicine.

Results The factors that have been found closely related to understand the role of health care personnel in EBM are Importance of realising the incorporation of EBM in day to day practice, The Role of health care personnel in generating relevant evidence, Structure of training of health personnel, Factors hampering the evidence synthesis, Choice of Evidence synthesis, role of clinical expertise and Leaders in Evidence Based medicine.

Conclusions The role models in EBM will require to get trained to be enlightened by the importance of EBM. The aim of bringing the clinicians on a platform to share a common goal of improvement in clinical practice, where they can address issues of obstacles in data acquisition, dissemination and implementation of evidence proven practice, administrative support provision as well as advances in the methods of improved accessibility of EBM.

\section{THE CREATION OF THE OXFORD-BRAZIL EBM ALLIANCE}

1,2,3 Luis Fontes, ${ }^{4,5}$ Rachel Riera, ${ }^{1,6}$ Regis Vieira, ${ }^{1,7}$ Rafael Pacheco, ${ }^{1,8}$ Ana Luiza Martimbianco, ${ }^{1,9}$ Daniela Pachito, ${ }^{10}$ Enderson Miranda, ${ }^{11}$ David Nunan. ${ }^{1}$ Universidade Federal de São Paulo (UNIFESP), São Paulo, Brazil; ${ }^{2}$ Faculdade de Medicina de Petrópolis, Petrópolis, Brazil; ${ }^{3}$ Department for Continuing Education, University of Oxford, Oxford, UK; ${ }^{4}$ Escola Paulista de Medicina (EPM) at Universidade Federal de São Paulo (UNIFESP), São Paulo, Brazil; ${ }^{5}$ Center of Health Technology Assessment, Hospital Sírio-Libanês, São Paulo, Brazil; ${ }^{6}$ Hospital Israelita Albert Einstein, São Paulo, Brazil; ${ }^{7}$ Centro Universitário São Camilo (CUSC), São Paulo, Brazil; ${ }^{8}$ Universidade Metropolitana de Santos, Santos, Brazil; ${ }^{9}$ Gestão e Economia de Saúde; Hospital Sírio-Libanês, São Paulo, São Paulo, Brazil; ${ }^{10}$ Centre for Evidence Based Medicine (CEBM), Department for Continuing Education, University of Oxford, Oxford, UK; ${ }^{11}$ Centre for Evidence Based Medicine (CEBM), Nuffield Department of Primary Care, Health Sciences, University of Oxford, Oxford, UK

\subsection{6/bmjebm-2019-EBMLive.96}

Objectives To describe the experience of building a teaching partnership between the Oxford CEBM and leading Brazilian Medical Schools, thus aiming to increase the effectiveness and quality of EBM teaching in Brazil.

Brazil is the 5th largest country in the world, with a population of approximately 210 million people. There are 336 active medical schools in Brazil, which graduate an average of 34,000 medical professionals each year. Of these schools, less than 20\% have in their curricula a formal Evidence-Based Medicine (EBM) discipline. The lack of formal EBM integration into medical school curricula is not exclusively a national challenge for Brazil. Data from the USA and UK suggests that only $60 \%$ of curricula include formal EBM content. Despite the recognition of the importance of EBM education and its influence on medical training around the world, challenges remain in integrating EBM education into the medical curriculum, facilitating communication between practitioners, increasing the capacity for EBM's meaningful use in clinical practice, and ensuring medical professionals have the knowledge and skills to support evidence-based practice. Concerned about these issues, two Oxford post-graduate students of EvidenceBased Health Care and a Senior Research Fellow of the Oxford Centre for Evidence-Based Medicine (CEBM) proposed the creation of a partnership between Oxford's CEBM and the EBM Departments of leading Brazilian medical schools. This partnership, named the 'Oxford-Brazil EBM Alliance,' aims to provide Evidence-Based Medicine and curriculum development training to medical educators (formal professors or tutors) in Brazil, thus fostering sustainability and replicability of effective teaching and learning throughout medical schools in Brazil. Objective: To describe the experience of building a teaching partnership between the Oxford CEBM and leading Brazilian Medical Schools, thus aiming to increase the effectiveness and quality of EBM teaching in Brazil. Method: Descriptive Case Study. Results: Two prominent Brazilian medical schools already joined the Oxford-Brazil EBM Alliance: Escola Paulista de Medicina at Universidade Federal de São Paulo (EPM-UNIFESP) and Faculdade de Medicina de Petrópolis (FMP-FASE). Training will be delivered via 3-day workshops, starting in June 2019, which will be facilitated by faculty from Oxford and Brazil. Other activities may emerge afterwards as instigated by local institutions. This project is underpinned in a concept of developing a 'Center of Excellence in EBM Teaching and Training', which intends to set the standard for EBM teaching in Brazil and throughout South America. It is our overarching aim that over time Brazil will have qualified faculty members to teach EBM in all its Medical Teaching Institutions. To our knowledge, a similar partnership does not exist in our region. In a second phase, we intend to extend this project to other countries in South America, offering further opportunities for international collaboration.

Method Descriptive Case Study.

Results Two prominent Brazilian medical schools already joined the Oxford-Brazil EBM Alliance: Escola Paulista de Medicina at Universidade Federal de São Paulo (EPM-UNIFESP) and Faculdade de Medicina de Petrópolis (FMP-FASE). Training will be delivered via 3-day workshops, starting in June 2019, which will be facilitated by faculty from Oxford and Brazil. Other activities may emerge afterwards as instigated by local institutions. This project is underpinned in a concept of developing a 'Center of Excellence in EBM Teaching and Training', which intends to set the standard for EBM teaching in Brazil and throughout South America. It is our overarching aim that over time Brazil will have qualified faculty members to teach EBM in all its Medical Teaching Institutions. To our knowledge, a similar partnership does not exist in our region. In a second phase, we intend to extend this project to other countries in South America, offering further opportunities for international collaboration.

Conclusions The Oxford - Brazil EBM Alliance is a promising partnership that can bring Evidence-Based Medicine training to thousands of medical professors and interdisciplinary health care teams throughout Brazil and its neighboring countries. The proposed 'teach the teacher' approach can facilitate EBM teaching and learning, foster future leaders, and ultimately benefit patients and healthcare systems throughout Brazil. 